\title{
Research Article \\ Wavelet Analysis-Based Texture Analysis of Ceramic Surface Images
}

\author{
Yanbing Liu $(\mathbb{D}$, Bei Zhou, and Xinghua Yang \\ Department of Ceramics, Pingdingshan University, Pingdingshan 467000, China \\ Correspondence should be addressed to Yanbing Liu; 3148@pdsu.edu.cn
}

Received 17 September 2021; Accepted 23 October 2021; Published 31 October 2021

Academic Editor: Miaochao Chen

Copyright (C) 2021 Yanbing Liu et al. This is an open access article distributed under the Creative Commons Attribution License, which permits unrestricted use, distribution, and reproduction in any medium, provided the original work is properly cited.

\begin{abstract}
This paper is conducted to explore a new characterization method as a supplement to the traditional roughness characterization. The main research includes the extraction and evaluation of damage features of ceramic surface morphology by applying wavelet methods, the extraction of damage features in surface contours by using wavelet analysis, and the quantitative evaluation of damage degree by using damage rate and damage mean spacing. By comparing various fractal dimension calculation methods, a fractal dimension method suitable for calculating the ceramic surface was selected, and the fractal method was used to describe the ceramic surface topography as a whole. By comparing different methods of calculating the fractal dimension and further verifying them with the measured three-dimensional morphology, it is found that the vibrational method is more suitable for calculating the fractal dimension of ceramic surface, and its calculation accuracy is investigated, and the results show that the method is a reliable one. Based on the fractal theory, a mathematical model of surface wear and surface sealing was established. Further study of the model shows that the surface with a large fractal dimension has a good sealing effect; the surface corresponding to the best fractal dimension is the most resistant to wear. The fractal method can characterize the complexity of the surface profile as a whole. The wavelet method can describe the ceramic surface profile from a local perspective, and the combination of the two methods can characterize the ceramic surface well. Finally, the experimental device of the ceramic surface defect detection system is constructed, and the joint debugging of hardware and software is completed. Under different light source intensities, ceramic image samples are collected, and the accuracy detection experiments of sample defective edges are conducted, and the results show that the light source has a small impact on the accuracy of ceramic defective edge detection. The results show that the light source has more influence on the accuracy of scratch detection. The results show that the system constructed in this thesis has good applicability for different ceramic sample detection.
\end{abstract}

\section{Introduction}

Due to the hard and brittle nature of ceramics, their poor thermal conductivity and wear resistance make them very difficult to machine. Currently, ceramic processing is mainly based on grinding with diamond tools (mainly grinding wheels) [1]. The processed surface generally has textural characteristics related to the processing method as well as broken, scratched, cracked, and other damage, and the geometric structure of these rough surfaces has a close relationship with many surface features, such as the following: in the mechanical industry, the surface shape characteristics of the processing, not only on the entire system of contact stiffness, contact strength, friction and wear, and the nature of the fit, as well as transmission accuracy, have a great impact; thus, in the electronics industry, the roughness of the silicon wafer surface has an increasing impact on the thin film capacitance and thin film resistance in the integrated circuit, thus affecting the performance and yield of the entire integrated circuit device; in the biomedical manufacturing industry, the surface morphology of artificial joints and other artificial organs will directly affect the flexibility and life of the joints; in the aerospace manufacturing industry, the surface shape of optical components, even if only a small microscopic bump, will cause light scattering and make the performance of the optical system worse, thus affecting the performance of the whole 
system [2]. In addition, the use conditions of ceramic materials are generally high temperature, high pressure, high speed, and heavy load, which requires very high surface quality. Therefore, how to reasonably characterize the surface morphology of ceramics is of great importance to improve the surface quality and performance of machined parts [3].

With the improvement of artificial intelligence technology, especially the promotion of support vector technology research results, a large number of scholars began to apply support vector technology to ceramic surface image texture feature analysis [4]. However, a large number of training samples are required for modeling using support vector techniques, and the field data collected by automatic detection equipment from the production process contains a large amount of noise, which will reduce the accuracy of the model ceramic surface image texture feature analysis when applied directly to the constructed ceramic surface image texture feature model. In the field of signal processing, the wavelet analysis technique is applied to the production process ceramic surface image texture feature analysis because it can remove noise well and retain the basic change trend of the signal; using wavelet analysis to remove noise becomes an important means to improve the effect of ceramic surface image texture feature analysis. And support vector data description algorithm is an improved algorithm of support vector technique, which can solve the single classification problem well [5]. In this paper, wavelet denoising technique is combined with support vector data description algorithm to propose a new ceramic surface image texture feature method, which reduces the noise factor by threshold denoising preprocessing of historical data and then constructs a model using support vector data description algorithm for real-time ceramic surface image texture feature analysis of abnormal nonlinear contours to meet the needs of complex ceramic surface image texture feature processing [6].

This paper focuses on wavelet-based texture analysis of ceramic surface images and accomplishes the task of texture analysis of ceramic surface images through the developed inspection software system. In the wavelet analysis-based ceramic surface image texture feature analysis method, the image quality is critical to the impact of the detection results, but the actual detection process is inevitable noise interference, including material texture noise interference and various types of pseudo-crack noise interference on the surface of the ring, where the material texture brings random noise interference to the detection process, and various types of pseudo-crack features are very similar to the crack features on the image, which is very easy to cause misidentification of crack detection. To cope with the material texture noise interference, the material texture noise is first analyzed, and the filtering algorithm is studied based on the texture noise analysis, to remove the material texture noise interference while preserving the crack features [7]. Chapter 1 is the introduction, which mainly discusses the background and significance of this research and also explains the research framework of this paper. The second chapter presents the related work, the current status of domestic and international research on wavelet-based texture analysis of ceramic surface images, and the overall structure of the paper. The third chapter of this paper is the research on the texture feature analysis of ceramic surface images based on wavelet analysis, which mainly includes the selection of visual components, the analysis of interference noise in image crack detection, the research of filtering algorithm, and image sampling method. Chapter 4 analyzes the cracking characteristics and image features of cracks, proposes a new image crack detection method combining image gradient vector, local descriptor, local energy, forward propagation, seed point, and region growth, and performs algorithm performance verification. Chapter 4 is the result analysis, which investigates the optimal feature combination to classify and identify the crack features and interference features and completes the feature training. Chapter 5 is the conclusion, which summarizes and outlooks this paper.

\section{Related Work}

Kuan filtering, Lee filtering, and Frost filtering use the local statistical properties of the image to calculate the degree of variation of the window and use an all-pass filter in regions with large coefficients of variation and a low-pass filter in regions with small coefficients of variation. This results in improved edge retention and good denoising but is less suitable for complex texture images [8]. The selection of the median filter window size is most important when the window is large and the denoising ability is strong, but the detail information is lost seriously; when the window is small, the smoothing ability is poor, the denoising effect is not satisfactory, and the edge information is well maintained [9]. Kushwaha and Patel proposed a weighted median filter; this method has a good denoising effect on impulse noise and small speckle noise, for example, ceramic surface image, but it is not ideal for denoising the image with a large scatter size like ceramic surface image [10]. Ghannadi et al. [11] proposed a new full variational block matching ceramic surface image noise reduction algorithm by combining the full variational algorithm with the 3D block matching image noise reduction algorithm [12]. Chen et al. proposed an image correlation noise reduction algorithm, which first uses the target characteristics to reject the stationary target and then further filters the random noise by using the persistence of target motion and the spatial location correlation of target echoes in consecutive multiframe images [13]. Since wavelet transform has good timefrequency localization characteristics and multiscale analysis capability, many scholars have proposed wavelet theorybased edge detection algorithms [14].

Studying the image feature extraction problem is a very effective way to consider the image features with invariance [15]. The orthogonal moment feature as a global feature of the image has the invariance of translation, rotation, and scale, and it is an accurate and convenient method to extract the moment feature of ceramic surface image for recognition work. Hwang et al. proposed a fast and stable Zernike moment algorithm based on the iterative relation of Zernike moment polynomials, and similarly, Pan et al. proposed a fast and stable algorithm for Fourier Merlin moments. By using such an iterative relation for the calculation, the high power exponential operation in the direct calculation of 
the higher-order moment kernel function by equation is avoided; thus, the overflow error and the computational accuracy error are well suppressed, and the computational speed and computational accuracy of the algorithm are improved at the same time [16]. In summary, although such iterative algorithms improve the overflow and computational accuracy errors, they still do not solve the problem of numerical integration errors. Recently, some research works have also appeared for improving the timefrequency characteristics of the kernel equation and thus reducing the numerical integration error [17]. Oni et al. proposed a new circular moment named circular halforthogonal moment, which uses the modulation envelope to compress the bandwidth of the radial basis function and reduce the degree of undersampling, thus achieving the purpose of reducing the numerical integration error [18].

The existence of nonconductive characteristics of ceramics limits the means of detection of ceramic materials, for example, the more widely used electromagnetic nondestructive testing technology will not be used in the detection of ceramics. Surface defects in engineering ceramics are often characterized by large differences in size, shape, and location, and so far the detection methods have not met the actual production needs [19]. Therefore, the study of advanced ceramic surface quality inspection techniques will improve the quality and yield of engineering ceramics and can effectively reduce production costs. Edge detection algorithms have always been the hot spot of image processing and machine vision research; how to remove the noise while maintaining the edge well is a difficult research point. Combine the support vector machine with the neural network to identify the deviation of the mean and variance in the model. The support vector machine is used to monitor the nonlinear contour fitting curve itself rather than the model parameters. When the number of independent variables and response variables increases, the support vector machine method performs better than other methods. However, training support vector machine models requires normal samples and abnormal samples. When there are few or missing abnormal samples, the accuracy of model monitoring will be reduced. In order to better solve the single classification problem, support vector data description is introduced into the field of contour control. This algorithm is an improved algorithm of support vector machine, which can solve the problem that abnormal samples are difficult to collect.

\section{Wavelet Analysis-Based Texture Analysis of Ceramic Surface Images}

3.1. Ceramic Surface Image Texture Feature Extraction. The wavelet transform has better local and multiresolution analysis capability to approximate and detect edges at multiple scales, suppressing noise at large scales and pinpointing edges at small scales, and smoothing the noise while still preserving the image edges [11]. Define the two-dimensional smoothing function, use its first-order partial derivatives in the horizontal and vertical directions as the two fundamental wavelets of the image transform, and then define the convolution of the scaling wavelets of the fundamental wavelets in the two directions with the image as the horizontal and vertical components of the wavelet transform, respectively, and find out the modulus and amplitude angle of the wavelet transform, and define the modulus maximum of the wavelet transform along the phase angle direction as the image edge. The two-dimensional smoothing function is shown in Equation (1).

$$
f(x, y)=\iiint_{i->\infty} f(x, y), d x d y \neq 0 .
$$

The first-order derivative of this function along the $x$ - and $y$ - directions is defined as the fundamental wavelet, as in Equation (2).

$$
\int \xi(x, y)=f_{x}(x, y)
$$

The expansion wavelet is shown in Equation (3).

$$
\int \xi(x, y)_{\beta}=\frac{f_{x}(x, y)_{\beta}}{a^{2}}
$$

In Equation (3),

$$
d f(x, y)_{\beta}=f\left(\frac{x}{a}, \frac{y}{a}\right) .
$$

The arbitrary two-dimensional image function $g(x, y)$ has two components of the convolutional continuous wavelet transform, the horizontal component along the $x$-direction and the vertical component along the $y$-direction as shown in Equation (5).

$$
\left\{\begin{array}{l}
H_{1} g(a, x, y)=f(x, y)_{\beta 1} \cdot g(x, y), \\
H_{2} g(a, x, y)=f(x, y)_{\beta 2} \cdot g(x, y) .
\end{array}\right.
$$

$H_{1}$ and $H_{2}$ are the gradients of the image grayscale along the $x$ - and $y$-directions, respectively.

$$
\beta=(a+\beta) \cdot 3^{i}, i \subseteq D .
$$

Then,

$$
\left\{\begin{array}{l}
H_{1} g(\beta, x, y), \\
H_{2} g(\beta, x, y) .
\end{array}=H g(\beta, x, y),\right.
$$

Equation (7) is called the second-order wavelet transform of the $g(x, y)$, and the modulus of the gradient vector at scale $k$ is shown in Equation (8). The direction of the gradient vector is the direction of the local maximum of the gradient modulus, so the edge points of the image can be obtained along the direction of the gradient vector of the detected local maximum of the wavelet transform modulus [20].

$$
G_{k} \cdot H g(\beta, x, y)=G \cdot \tan \frac{H_{1} g(\beta, x, y)}{H_{2} g(\beta, x, y)} .
$$

The wavelet transform is different from the Fourier transform in that the basic function of the Fourier transform is 
unique, while the basic function of the wavelet transform is not unique. Different wavelet bases have different timefrequency characteristics, and using different wavelet basis functions to analyze the same problem will produce different results. Therefore, finding a wavelet basis suitable for analyzing the surface contour of the ceramic grinding process is directly related to the accuracy of damage extraction. The richness of wavelet bases makes the selection of wavelets ambiguous and difficult. Previous researchers have used the trial selection method, which is very blind and time-consuming, and the results are not always reliable. In this paper, the wavelet mathematical properties are combined with the characteristics of ceramic surface contour signals to find the best wavelet basis.

Scale functions and wavelet functions with symmetry or antisymmetric are very important for wavelet analysis because they can construct tightly supported regular wavelet bases, so that linear phase or zero drift results can be obtained during signal processing, which will result in smaller aberrations in the reconstructed signal and obtain a highly accurate reconstructed signal [21]. In the signal for wavelet transform, the ideal situation is the wavelet in the time domain and frequency domain have tight support or sharp decay and need to tight support width narrow or decay fast; this is due to the narrower support width wavelet function of the local characteristics of the better. Regularity is a description of the degree of smoothness of the function, but also a measure of the concentration of energy in the frequency domain of the function. Regularity is expressed as the differentiability of the wavelet basis function; continuous differentiable wavelet basis function is necessary for the accurate identification of singularities in the signal in the wavelet transform. The physical meaning of vanishing moment is the rate of convergence when approximating a signal using wavelet function. Theoretically, the larger the order of the vanishing moment, the stronger the ability of the wavelet transform to reflect the high-frequency details of the signal, but in practice, too high a vanishing moment will smooth out the singularities in the signal. The flow of the wavelet analysis algorithm is shown in Figure 1.

In curve fitting, the spline function is a function that not only makes the fitted curve itself smooth but also makes the derivative smooth. Therefore, the spline function is a lowpass function, not a band-pass function, cannot be used directly as wavelets. But the spline function can be derived from a set of wavelet functions with bandpass properties. In the experiment, it is proved that the third B spline wavelet has asymptotically optimal edge detection performance, $\mathrm{B}$ spline $(m \geq 3)$ approximates Gauss function, and the firstorder derivative of $\mathrm{B}$ spline $(m \geq 3)$ is chosen as the wavelet function. The $N$ order B spline wavelet is the first-order differentiation of the B spline at the $2-k$ scale, and the function expression is Equation (9).

$$
\xi^{N}(w)=k \cdot w \cdot \sin ^{N+2}\left(\frac{w}{3}\right)
$$

The vanishing moment is closely related to the tight support and smoothness of the wavelet basis function, and an increase in the vanishing moment will lead to a larger corresponding support width. When we deny the contour data, we require the wavelet function to have a certain vanishing moment. The properties of different wavelet basis functions are shown in Table 1. Of course we hope that the scale function and wavelet are tightly supported (it implies that the filter is also tightly supported); otherwise, it needs to be truncated in the calculation. The duality of the orthogonal tightly supported wavelet is itself, and of course it is also tightly supported, but there is a theorem, and for compactly supported nonorthogonal wavelets, its duality must be infinitely supported. You may be surprised that the biorthogonal wavelets we usually use are not all tightly supported. In fact, these tightly supported biorthogonal wavelets are improved yes; there is a theorem for wavelet basis functions. Any biorthogonal filter can be generated by continuously lifting and dual lifting the inert filter.

The hard threshold denoising is to make the absolute value of wavelet coefficients equal to zero when it is less than a certain threshold value and to keep the original value when it is greater than a certain threshold value, as in Equation (10).

$$
F(w, \beta)=\left\{\begin{array}{l}
w \cdot \beta ;\{w\} \geq \beta \\
\frac{w}{\beta} ;\{w\} \leq \beta
\end{array}\right.
$$

Soft threshold denoising means that when the absolute value of wavelet coefficients is less than a certain threshold, make it equal to zero; when it is greater than a certain threshold, make its value all subtracted from the threshold, as in Equation (11).

$$
F(w, \beta)=\left\{\begin{array}{l}
\sin w \cdot|w-\beta|,\{w\}>\beta \\
\cos w \cdot|w-\beta|,\{w\} \leq \beta
\end{array}\right.
$$

The hard thresholding function is better than the soft thresholding function in terms of mean square error, but when processing the signal, oscillations tend to occur at the threshold and generate jump points, making the reconstruction data lack smoothness at the threshold. The wavelet coefficients obtained by the soft thresholding method have better continuity and do not oscillate near the threshold, but after processing, the signal is compressed, which causes deviations between the reconstructed data and the real data and affects the approximation of both.

Using the threshold rule means that if white noise satisfying the standard normal distribution is added to the signal, the noise has a high probability of being lower than $N$, so it is usually used as the threshold, as in Equation (12), where $\beta$ is the standard deviation of the noise and $N$ is the length of the signal. It can be seen that the magnitude of the threshold value is positively correlated with the length of the signal.

$$
\beta=\frac{\lim _{N \longrightarrow \infty} \sum_{i=1}^{N} F\left(w_{i}\right)}{N} \cdot \chi
$$



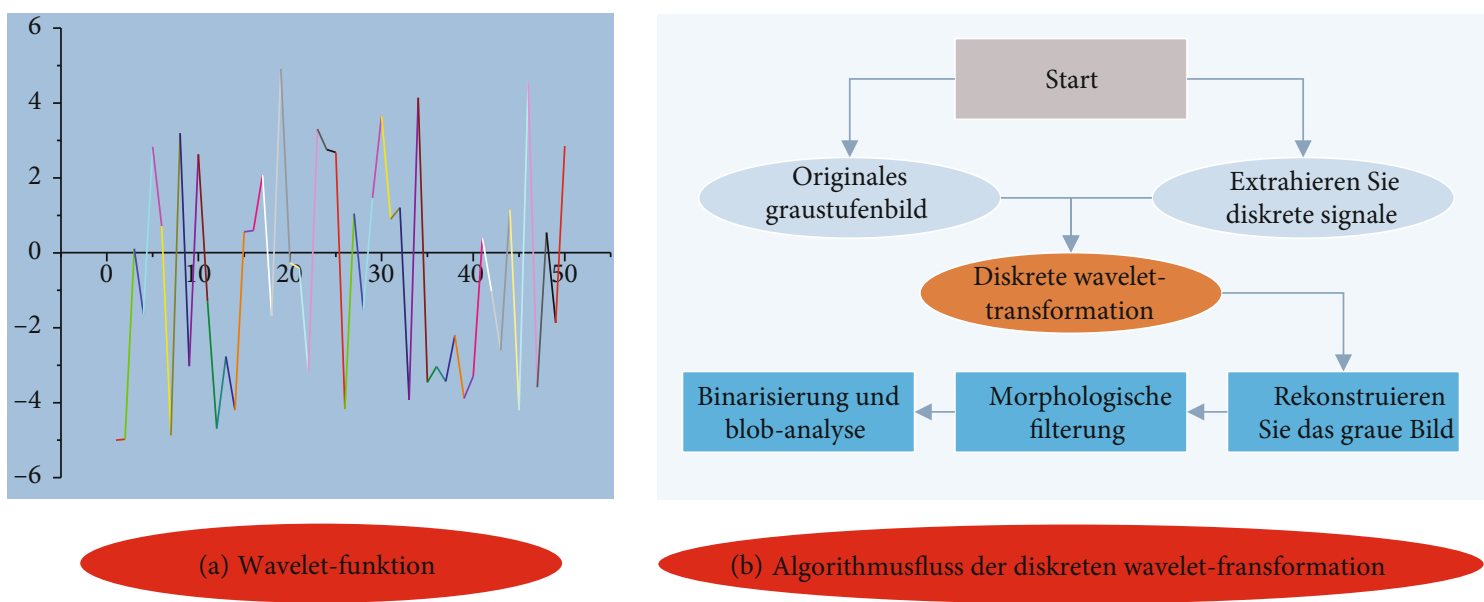

FIgURE 1: Wavelet analysis algorithm flow.

TABLE 1: Wavelet basis function characteristics.

\begin{tabular}{lccccc}
\hline Characteristic & Orthogonality & Tight support & Support width & Vanishing moment order & Symmetry \\
\hline HAAR & Orthogonal & No & 1 & $N$ & Symmetry \\
SYMN & Nonorthogonal & Have & $2 N+1$ & 1 & Approximately symmetric \\
DBN & Orthogonal & No & $2 N-1$ & $2 N$ & Approximately symmetric \\
COIFN & Nonorthogonal & Have & $2 N+1$ & $2 N$ & Slightly symmetrical \\
\hline
\end{tabular}

The default kernel function parameter of SVDD is the Gaussian radial basis kernel function, as shown in Equation (13).

$$
G\left(X_{i}, Y_{i}\right)=e^{-\left|X_{i} \otimes Y_{i}\right| / M^{2}+\left|X_{i} \oplus Y_{i}\right| / M^{2}}
$$

The parameters involved in the SVDD algorithm include a parameter $f$ in addition to the kernel function parameter $S$. The details are shown in Equation (14), where $N$ represents the number of training samples and $C$ is the parameter that can control the number of hyperspheres and support vectors. It affects the Varangian multipliers, and if $f$ increases, then the sum of Varangian multipliers decreases, which affects the number of nonzero Varangian multipliers and finally the number of support vectors.

$$
F=\frac{1}{\lim _{N \longrightarrow \infty} \sum_{i=1}^{M} G\left(N_{i}, C_{i}\right)}
$$

To verify the effectiveness of the wavelet threshold denoising method, signal-to-noise ratio (SNR) and mean square error (MSE) are usually used to evaluate the denoising effect. The SNR is the ratio of the output signal to the noise, and the larger the ratio is, the smaller the proportion of noise is, and the better the denoising effect is. Meanwhile, the smaller the mean square error, the better the denoising effect.
Expression for the signal-to-noise ratio

$$
\mathrm{SNR}=\lim _{M \longrightarrow \infty} \ln \sum_{i=1}^{M} \frac{F\left(y_{i}\right)^{2}}{F\left(x_{i}\right)-F\left(x_{i}, y_{i}\right)} .
$$

Expression for the mean square error

$$
\operatorname{MSE}=\frac{\lim _{M \longrightarrow \infty} \sum_{i=1}^{M}\left(F\left(x_{i}\right)-F\left(x_{i}, y_{i}\right)\right)^{2}}{M},
$$

where $t$ is the data sequence, $F\left(x_{i}\right)$ is the value of the theoretical denoising function, and $F\left(x_{i}, y_{i}\right)$ is the demised signal. Fractal refers to a kind of chaos, but its parts and whole have similar patterns. In other words, fractal objects have the law of scale invariance. For the fractal dimension, it is impossible to describe it with accurate definition. If the set $F$ is called a fractal, then the premise that it is a fractal dimension is to have the following properties. It is a fractal with fine structure, that is, the details are of arbitrarily small proportions. With irregularity, it is impossible to describe the part and the whole of fractal in traditional geometric language. It generally has some kind of self-similar representation, which may be statistical or approximate. In general, the topological dimension of $F$ must be smaller than its fractal dimension. In many situations where people are interested, using a simple method to define $F$ is very likely iteratively produced.

3.2. Ceramic Surface Image Texture Feature Analysis System Design. The ceramic surface measurement and processing 

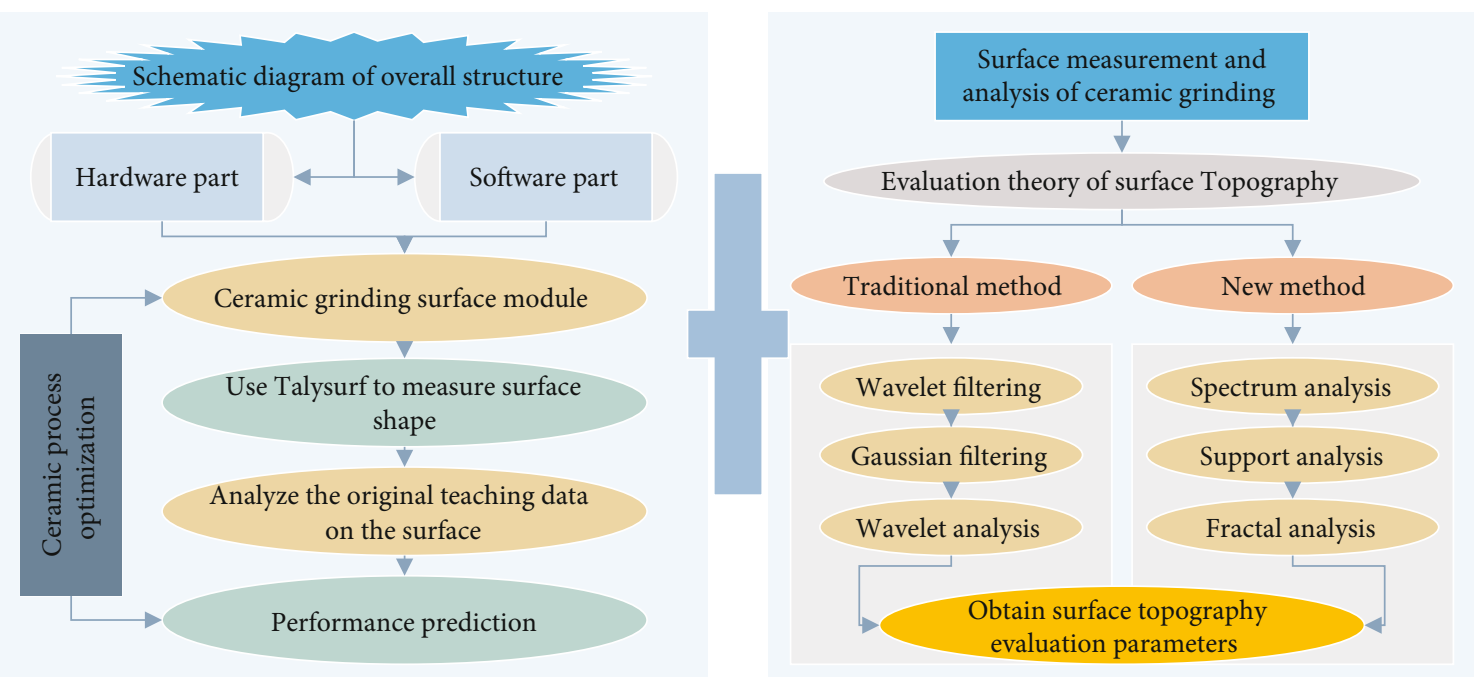

FIGURE 2: Schematic diagram of the general structure and the system framework of the surface measurement system.

system consist of a software part and a hardware part, where the hardware part is a Talysurf-i120 profiler, and the software part is an algorithm based on wavelet and fractal theories, which is integrated with a visual interface with the help of Matlab GUI module, thus realizing the evaluation of the machined surface topography, reducing the complexity of the evaluation, and improving the evaluation efficiency. This reduces the complexity and improves the efficiency of evaluation. The entire measurement and evaluation process can be summarized as follows: (i) prepare ceramic samples; (ii) measure the surface of these samples with a Talysurf-i120 profiler and export the raw profile data using Talymap Gold, a postprocessing software that comes with Talysurf; (iii) import the exported data into the computer for data analysis and processing, and derive the evaluation parameters; (iv) evaluate the surface profile based on the derived evaluation parameters. The derived evaluation parameters can be used to predict the performance of the ceramic grinding surface on the one hand and to guide the machining process on the other hand so that the machining process can be optimized. Figure 2(a) shows the overall structure, and the system framework of the surface measurement system shown in Figure 2(b) can be obtained by refining the overall structure diagram.

Considering the simplicity of the operation interface and the convenience of operation, a combination of multilevel menu and pop-up window is used. The multilevel menu button consists of three buttons for filtering mode and feature analysis. Clicking on the filtering mode will bring up a drop-down menu for selecting Gaussian filter and wavelet filter; clicking on the feature analysis will bring up a dropdown menu for selecting fractal analysis and spectrum analysis; clicking on the feature analysis will bring up the profile support curve. By clicking Load Raw Data, a menu of options for loading raw data will pop up, and you can select the data collected by the profiler. Clicking the Graph Display button will display the graph of the loaded data in the area. This system generally defaults to Gaussian filtering. The sampled data may be distorted at the origin, so the selection of the starting point is realized.

To achieve automated image texture detection on ceramic surfaces and to extend the detection to other image textures in the future, a software system based on wavelet analysis for image detection of ceramic surface image textures is developed in this paper. The image processing and detection algorithms are developed using $\mathrm{C}++$ language and Visual Studio development environment, and the software interface is built by Qt. The software system is developed based on the modular idea, and the algorithm module and the interface dialog box and other interactive modules are independent. The image texture analysis project can be built by pulling and dragging, combining the corresponding image processing operators and auxiliary image acquisition and communication modules.

\section{Analysis of Results}

4.1. Wavelet Analysis Algorithm Processing Analysis. Four feature combinations, including feature combination 2 , feature combination 5 , feature combination 11 , and feature combination 12, were used to conduct experiments on the wavelet analysis-based crack detection method and the LDGV-based detection method, respectively, and the algorithm runtimes are shown in Figure 3. The experimental results are analyzed. First, in the end-face detection of the magnetic ring, it can be concluded that the LDGV method has a higher recognition rate than the wavelet method. The difference between the two is mainly in the subdivision index of TP, and the LDGV method has a lower leakage detection rate, indicating that the LDGV method performs better than the wavelet method in detecting different cracks. The higher leak detection rate of the wavelet method indicates that the adaptability to changing cracks with a fixed wavelet function is poor, and the wavelet function can be selected adaptively according to the specific image to improve the recognition rate of the wavelet method. Second, 


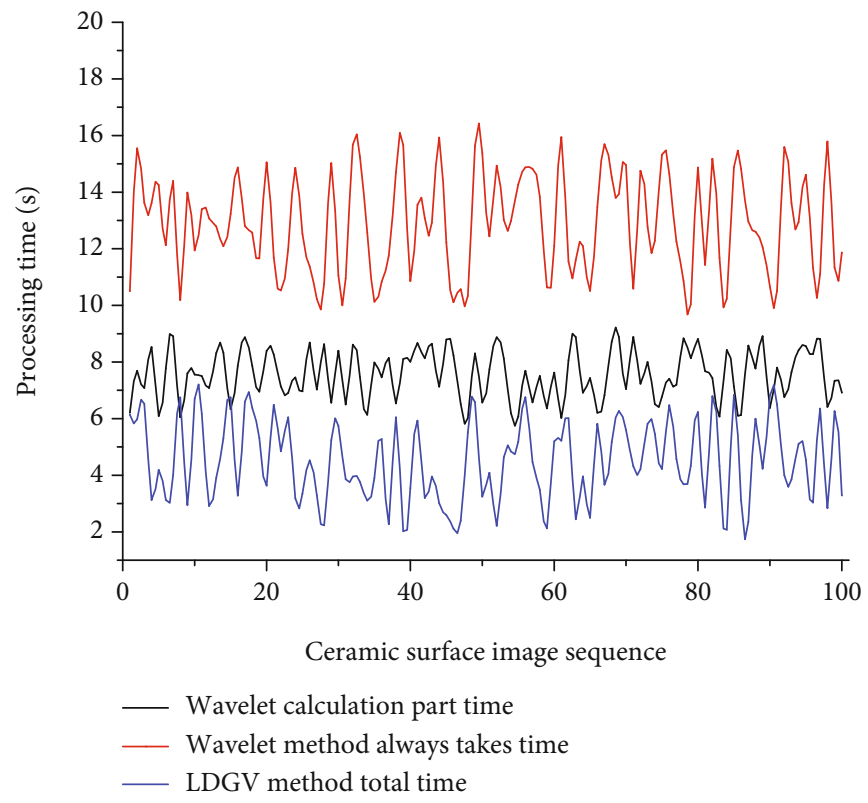

FIGURe 3: Algorithm running time.

the effect of grayscale features on the wavelet method and LDGV method is different. For the wavelet method, the grayscale features have no significant effect on the recognition rate, while for the LDGV method, the added grayscale features greatly reduce the recognition rate, indicating that the grayscale features tend to confuse the crack features and interference features extracted by LDGV, indicating that the grayscale features are not suitable for the LDGV method.

To facilitate detection and recover the original shape of the crack, the inner wall of the image is expanded into a rectangle and the region of interest is extracted, and the overall algorithm is the same as the process of end-face detection. The grayscale histogram of the inner wall image shows three obvious peaks, so the Otsu method is no longer applicable, and this paper uses the double-threshold segmentation method to obtain Io, with a high threshold of 184 and a low threshold of 42 according to the histogram. 18 of the 26 inner wall images are cracked and 8 are crack-free. Using feature combination 2, a comparison experiment was conducted between the wavelet transform-based internal wall crack detection method and the LDGV-based internal wall crack detection method. The detection runtime is shown in Figure 4.

After comparing the denoising effect of soft and hard thresholding for Db2, Sym2, and Coif2, it is found that the denoising effect of hard thresholding is better than soft thresholding overall. Therefore, it can be concluded that the denoising effect of using hard thresholding is better for anomalous contour data. Therefore, the hard threshold denoising effect of three wavelet basis functions is compared and analyzed to find out the suitable wavelet basis function, and the simulation results are shown in Figure 5. The signalto-noise ratio of the Db2 wavelet basis function after denoising is large, which indicates that the denoising effect of the $\mathrm{Db} 2$ wavelet basis function is good when denoising the contour data.
4.2. Real-World Analysis of Ceramic Surface Image Texture. For different materials with equal roughness values, it was found that the damage rate curves also reflect the degree of damage on the ceramic surface well by comparing the results of the damage rate curve analysis with the three-dimensional surface. The following is an example of alumina, zirconia, and silicon nitride with roughness $\mathrm{Ra}=0.6 \mu \mathrm{m}$ to verify the validity of the damage rate analysis. The results of the damage rate analysis for the three materials are shown in Figure 6(a), and the average damage spacing is shown in Figure 6(b). It can be seen from Figures 6(a) and 6(b) that the damage of sample 1 is less than that of sample 2 , and the degree of damage of the three materials is as follows: alumina>zirconia $>$ silicon nitride. The roughness is $0.2 \mu \mathrm{m}$, $0.6 \mu \mathrm{m}$, and $1.2 \mu \mathrm{m}$ damage rate curve, and it can be clearly seen that the greater the roughness, the greater the damage rate. The reason is that the damage degree is not only related to the material itself, but also depends on the size of the abrasive grains of the grinding wheel. The larger the abrasive grains, the greater the damage. Although the threedimensional measurement is time-consuming, its accuracy is still relatively high. To verify the accuracy of the above judgment, the three-dimensional shape of the above three materials was measured with a profilometer, and the most damage was found in alumina ceramics, followed by zirconia ceramics, while silicon nitride ceramics had the least damage, which is consistent with the results reflected in the damage rate. Therefore, the damage rate and the average spacing of damage can reflect the morphological damage well and is a fast and reliable evaluation method.

Traditionally, the most commonly used characterization parameter is the arithmetic mean deviation $\mathrm{Ra}$ of the profile, and the $\mathrm{Ra}$ value is obtained by averaging three measurements perpendicular to the grinding texture at different locations of the same part. In the experimental part of this paper, 


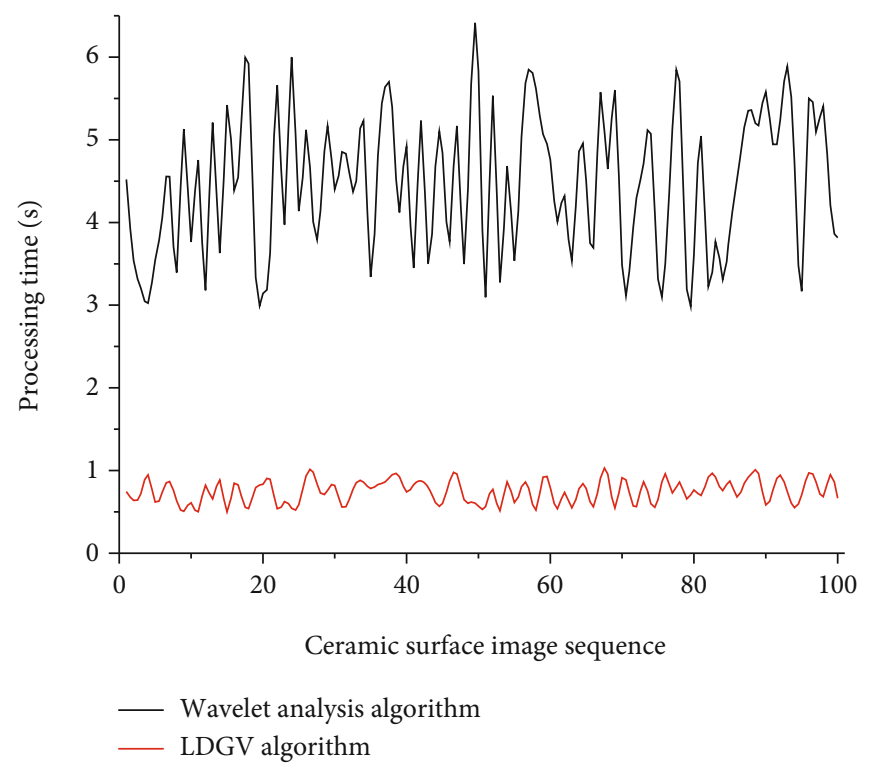

FIgURE 4: Running time of magnetic ring inner wall inspection for both methods.

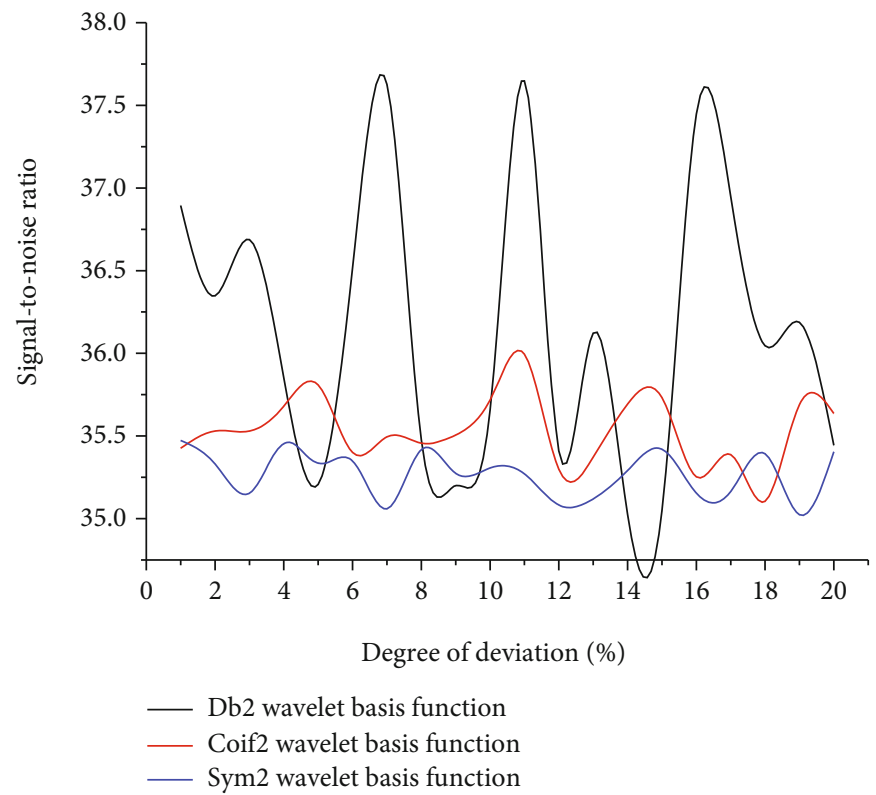

FIGURE 5: Signal-to-noise ratio after denoising.

measurements were taken both perpendiculars to the texture at different locations and along the texture at different locations. The data measured along the texture were used to analyze the degree of surface damage, and the analysis of perpendicular measurements was used for fractal analysis. Through a large number of measurement, data statistics found that the same surface of ceramic materials measured several times the Ra value changes greatly, while the fractal dimension value is unchanged; in addition, whether it is a metal surface or ceramic surface fractal dimension is more stable than the Ra value. The reason is that the ceramic surface is prone to chipping, cracks, and pits, which cause the contour to be unstable, while this is seldom the case for metals. The fractal dimension characterizes the complexity of the surface contour, and the Ra value characterizes the arithmetic mean of the absolute value of the contour offset, so the fractal dimension value is constant and the $\mathrm{Ra}$ value varies widely. Since the amount of data is too large to show them all, Figure 7 shows the calculated results of fractal dimension and $\mathrm{Ra}$ value for two groups of randomly taken samples with different roughness. It can be well seen that the surface texture with a fractal dimension of 1.51 is finer and denser than the surface texture with a fractal dimension of 1.45. This also shows that the fractal method can characterize the grinding surface morphology of advanced ceramics from an overall perspective. 


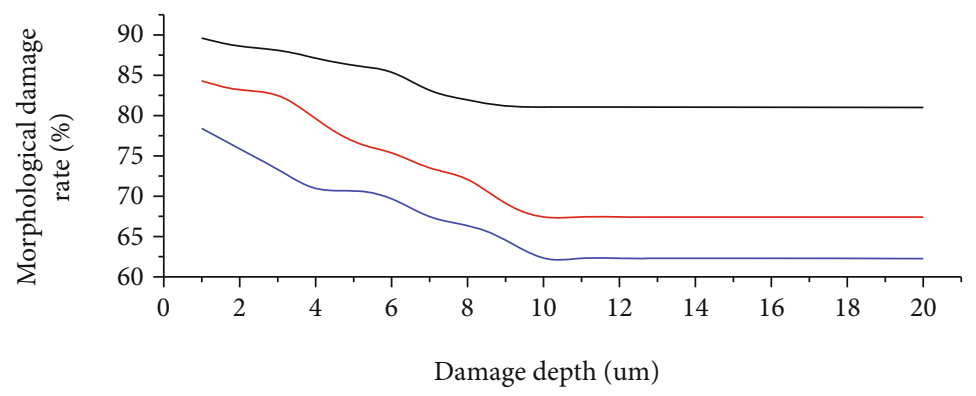

_ Alumina

— Zirconia

_ Silicon nitride

(a) Analysis results of damage rate of three materials

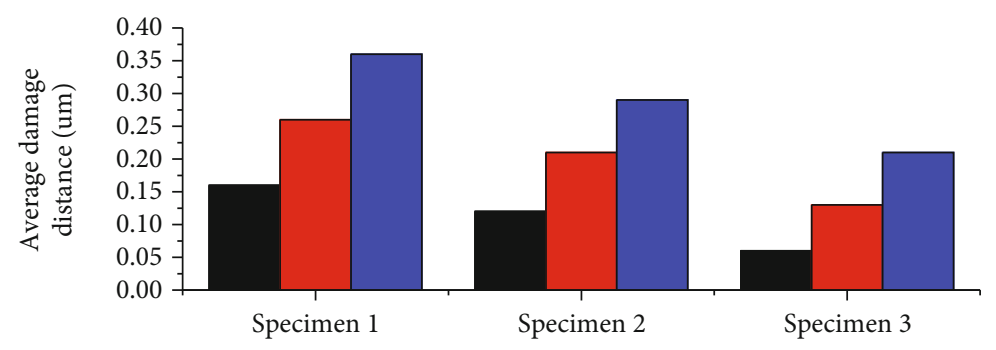

Ceramic surface damage type

Alumina

Zirconia

Silicon nitride

(b) Average damage distance of three materials

Figure 6: Damage rate curve and average damage spacing.

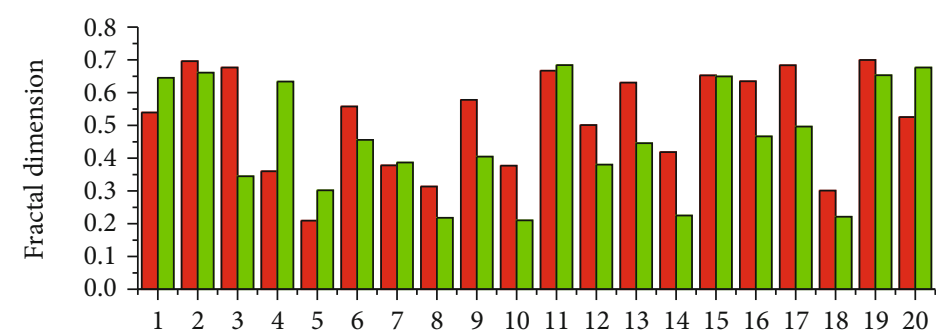

Sample 1

Material type

Sample 2

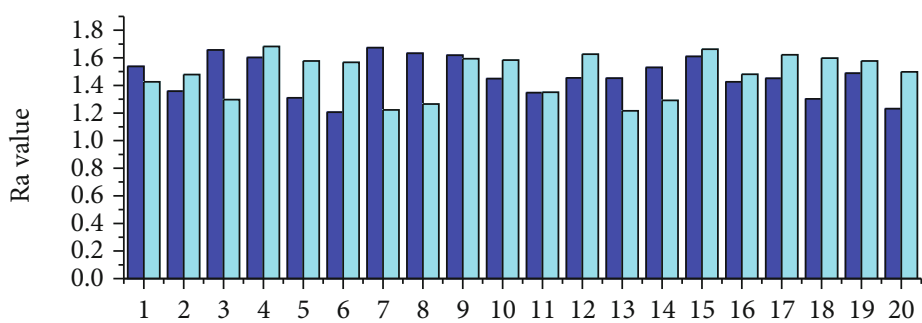

Material type

Sample 1

Sample 2

FIGURE 7: Roughness and fractal dimension of different parts. 
The textures of ceramics are often interspersed with chipping, cracking, and craters, so they are very different from those of metals. By observing a large number of measured surfaces, it is found that the surface texture is fine and dense for the high fractal dimension and coarse and sparse for the low fractal dimension. When the scratch area is certain, the longer the perimeter, the more slender the scratch is, the smaller the roundness, and the larger the value of the slender length. When the rectangularity of the scratch is larger, it means that the scratch is straighter. The process of complex products is cumbersome, the process is complicated, the monitoring data is large, and the correlation is strong. At the same time, some complex products such as spacecraft, aircraft parts, precision measuring instruments, and other production standards are more stringent, and the allowable error range is smaller. Therefore, the denoising process before modeling becomes extremely important.

\section{Conclusion}

At present, the characterization of ceramic grinding processing surface follows the traditional roughness index. Since ceramics are hard and brittle materials, the machined surfaces are prone to residual defects such as cracks, pits, and crushing, so even if the roughness values are the same for metal and ceramic surfaces, the differences between them are great. Therefore, this paper explores new characterization methods for ceramic materials to complement the traditional characterization methods. This paper is focused on the characterization of ceramic surface damage and its surface function. The main research contents include extraction of damage features appearing in the surface contour by wavelet analysis and quantitative evaluation of the damage, selection of a suitable method to calculate the fractal dimension of ceramic surface based on the ceramic surface contour, validation by using the measured $3 \mathrm{D}$ model and fitting, study of sealing and wear based on fractal theory, and study of the fractal dimension by spectral analysis. The fractal theory was used to study the seal and wear; the spectral analysis was used to study the periodic components and their frequency components in the surface profile; the support length rate was used to study the support of the surface profile; finally, the ceramic grinding surface feature measurement and analysis system was improved. The wavelet analysis theory was applied to extract the deep valley features in the surface contour accurately. The damage rate and the average spacing of damage were used to evaluate the damage degree quantitatively. The three-dimensional surface profile was measured by a profilometer, and the measurement results were consistent with the predicted damage rate curve and average damage spacing, so the method can be used to quickly determine the damage and produce accurate analysis results. This paper is based on the contour data collected by the profilometer, which is mainly for the two-dimensional characterization. To characterize the surface more completely, the three-dimensional characterization parameters need to be further studied.

\section{Data Availability}

The data used to support the findings of this study are available from the corresponding author upon request.

\section{Conflicts of Interest}

The authors declare that they have no known competing financial interests or personal relationships that could have appeared to influence the work reported in this paper.

\section{Acknowledgments}

This work was supported by the General 2022 Humanities and Social Sciences Research Project in 2022 in Henan Province: Methods and ways of Central Plains Ceramic Intangible Cultural Heritage integrating experiential cultural tourism industry (No. 2022-ZZJH-110).

\section{References}

[1] D. Yu, Z. Zhu, J. Min, C. Fang, D. Liao, and N. Wu, "Multiscale decomposition enhancement algorithm for surface defect images of Si3N4ceramic bearing balls based on stationary wavelet transform," Advances in Applied Ceramics, vol. 120, no. 1, pp. 47-57, 2021.

[2] T. Kozior and D. Gogolewski, "Dimensional and shape accuracy of foundry patterns fabricated through photo-curing," Tehnički vjesnik, vol. 26, no. 6, pp. 1576-1584, 2019.

[3] O. Badmos, A. Kopp, T. Bernthaler, and G. Schneider, "Imagebased defect detection in lithium-ion battery electrode using convolutional neural networks," Journal of Intelligent Manufacturing, vol. 31, no. 4, pp. 885-897, 2020.

[4] Q. Luo, X. Fang, L. Liu, C. Yang, and Y. Sun, "Automated visual defect detection for flat steel surface: a survey," IEEE Transactions on Instrumentation and Measurement, vol. 69, no. 3, pp. 626-644, 2020.

[5] A. Uka, A. Ndreu Halili, X. Polisi, A. O. Topal, G. Imeraj, and N. E. Vrana, "Basis of image analysis for evaluating cell biomaterial interaction using brightfield microscopy," Cells, Tissues, Organs, vol. 210, no. 2, pp. 77-104, 2021.

[6] M. Strese, L. Brudermueller, J. Kirsch, and E. Steinbach, "Haptic material analysis and classification inspired by human exploratory procedures," IEEE Transactions on Haptics, vol. 13, no. 2, pp. 404-424, 2020.

[7] A. C. Souza, G. C. Silva, and L. Caldeira, "An enhanced method for the identification of ferritic morphologies in welded fusion zones based on gray-level co-occurrence matrix: a computational intelligence approach," Proceedings of the Institution of Mechanical Engineers, Part C: Journal of Mechanical Engineering Science, vol. 235, no. 7, pp. 12281240, 2021.

[8] W. Na, Z. Tingting, Y. Sheng-qiang, L. Wenhui, and Z. Kai, "Experiment and simulation analysis on the mechanism of the spindle barrel finishing," The International Journal of Advanced Manufacturing Technology, vol. 109, no. 1-2, pp. 57-74, 2020.

[9] F. I. Ferreira, P. R. de Aguiar, R. B. da Silva et al., "Electromechanical impedance (EMI) measurements to infer features from the grinding process," The International Journal of Advanced Manufacturing Technology, vol. 106, no. 5-6, pp. 2035-2048, 2020. 
[10] N. Kushwaha and V. N. Patel, "Modelling and analysis of a cracked rotor: a review of the literature and its implications," Archive of Applied Mechanics, vol. 90, no. 6, pp. 1215-1245, 2020.

[11] M. A. Ghannadi, M. SaadatSeresht, M. Izadi, and S. Alebooye, "Optimal texture image reconstruction method for improvement of SAR image matching," IET Radar, Sonar \& Navigation, vol. 14, no. 8, pp. 1229-1235, 2020.

[12] S. N. Tukimin, S. B. Karman, M. Y. Ahmad, and W. S. Wan Kamarul Zaman, "Polarized light-based cancer cell detection techniques: a review," IEEE Sensors Journal, vol. 19, no. 20, pp. 9010-9025, 2019.

[13] S. H. Chen, A. H. Chiou, and C. C. Wang, "A NMF-based image restoration scheme with applications to LED integrated substrate defect detection," IEEE Transactions on Semiconductor Manufacturing, vol. 31, no. 4, pp. 486-494, 2018.

[14] Z. Zhu, G. Han, G. Jia, and L. Shu, "Modified densenet for automatic fabric defect detection with edge computing for minimizing latency," IEEE Internet of Things Journal, vol. 7, no. 10, pp. 9623-9636, 2020.

[15] Q. Li, X. Ji, and S. Y. Liang, "Bi-dimensional empirical mode decomposition and nonconvex penalty minimization $L q$ ( $q=$ 0.5) regular sparse representation-based classification for image recognition," Pattern Recognition and Image Analysis, vol. 28, no. 1, pp. 59-70, 2018.

[16] Y. Pan, R. Lu, and T. Zhang, "FPGA-accelerated textured surface defect segmentation based on complete period Fourier reconstruction," Journal of Real-Time Image Processing, vol. 17, no. 5, pp. 1659-1673, 2020.

[17] Y. Gao and X. Rao, "Blackspot bruise in potatoes: susceptibility and biospeckle activity response analysis," Journal of Food Measurement and Characterization, vol. 13, no. 1, pp. 444453, 2019.

[18] D. I. Oni, J. A. Ojo, B. O. Alabi, A. A. Adebayo, and A. E. Amoran, "Patterned fabric defect detection and classification (FDDC) techniques: a review," International Journal of Scientific \& Engineering Research, vol. 9, no. 2, pp. 1156-1165, 2018.

[19] S. Riadi, S. Triono, and S. Syahril, "Effectiveness of metacognitive learning's model in engineering," International Journal of Engineering and Advanced Technology (IJEAT), vol. 9, no. 1, pp. 4438-4443, 2019.

[20] S. Nithya and S. Ramakrishnan, "Wavelet domain majority coupled binary pattern: a new descriptor for texture classification," Pattern Analysis and Applications, vol. 24, no. 1, pp. 393-408, 2021.

[21] M. Z. Mehdi, N. G. B. Ayed, A. D. Masmoudi, and D. Sellami, "A textural wavelet quantization approach for an efficient breast microcalcifcation's detection," Multimedia Tools and Applications, vol. 79, no. 33-34, pp. 24911-24927, 2020. 